\title{
Violence Resisting Transforming Robot with Ability of Obstacle Detection
}

\author{
Bishal Karmakar
}

\begin{abstract}
This paper presents the design; Mechanism and implementation of Automatic Law enforce Transforming Robot with the ability to resist violence automatically or manually as instructed by the operator. The robot also contains a specialty of detection of protesters by sonar technique before activating water thrower system. The robot consists of two hands and one of those is carrying water thrower system. Any kind of anti-violence devices can be added in these two hands. With this, any violence can be cool down or any internal attack can be turned down which eventually is hard and dangerous for law enforce officers. For its transforming structure, it can easily be transported, helpful to use in both mode and can be addressed as non-threatening design for public. Arduino Uno performs as the brain that controls the whole body structure.
\end{abstract}

Index Terms-Arduino UNO; HS-5805 Mega Servo; SG90 Micro Servo; HC-SR 04 Sonar Sensor; L293D DC Motor Driver IC; Water Pump.

\section{INTRODUCTION}

A robot is a mechanical intelligent executor which can operate tasks on its own, or with instructions. Theoretically and practically a robot is an electro-mechanical machine which is handled by the computer and electronic programming. Robots can be addressed as autonomous or semi-autonomous type. The autonomous robot can be referred as the robots which are used for research into human-like systems, such as ASIMO and TOPIO [1]. The main tendency of this kind of autonomous robots is to shorten human efforts, time, life risk, error due to human thoughtlessness and bringing perfection. Using automation technology in the area of law enforcers, it is possible to use robots freely and work on difficult areas which are either inaccessible or dangerous for law enforce officers [2].For example, US police had operated a robot in a show of fatal force. Dallas police used a bomb-disposal robot carrying an explosive device on its manipulator's arm to execute a suspect after five police officers were shoot down [3]. Law enforcement is a life-threatening activity. This fact creates an acceptable field for robots. It is, anyhow, highly disorganized and that proves it very problematic for robots. Robots have launched the application in training, surveillance and resisting violence [4].

In this paper, a robot that can be used in the field of law enforcement is introduced. More specific it can be used to resist protests or violence. It can be operated in both automatic and manual mode. In automatic mode, the robot can be transformed from car shape into an approximate human form according to the logic. It has a water thrower system which works as a resisting device. It uses two logics to activate and work. If a group of protester comes under first logic it transforms and activates resisting device. If a group of protester comes under second logic it aback a predefined distance and then transform and activate resisting device. It consists of two mega servos and four micro metal servos for transformation. First mega servo brings the whole body behind by rotating $180^{\circ}$ and the second mega servo helps to create an approximate human form with resisting device by rotating $180^{\circ}$. The first two micro servos are used for opening and closing the hands and among them, one is carrying a water thrower system. Second two micro servos are used to give supports for the whole transformed robot. Two dc motors are used for the movement of the robot and a water pump is used for the water thrower system. These whole systems depend on the ultrasonic sensor. Two predefined logics are defined for the sensor. For simplicity of operation and minimization of cost, locally available components, and scraps were used to make the body [2]. To control the motors, servos, pump, and sonar sensor Arduino UNO is used. This is basically a microcontroller with 32 bit. This Arduino's model consists of 13 PWM pins and among which 9 are needed for controlling the selected components. The operating signals are given by the Arduino according to the response of sonar sensor. Such a device can be used for resisting the protests which are difficult and dangerous for law enforces's officers.

\section{LITERATURE REVIEW}

K.R.Chaubey, T.Singh, S.Jasuja, in their paper proposed a model that can transform leg to wheel or wheel to leg. One of two disadvantage of their work is that their robot can't transform the whole body. And another massive disadvantage is their robot only can operate in manual mode, therefore, it can't make self-decision [5].

The proposed system is not only an improved version but also comes with an additional feature that helps the robot to fit in its targeted place. This modified system can transform with the whole body and have a self-decision making technique. With its additional feature, it is suitable to use in law enforce field.

Published on December 16, 2016.

Bishal Karmakar is from Department of Electrical and Electronic Engineering, Varendra University, Rajshahi, Bangladesh. (bishal.karmakar95@gmail.com). 


\section{SYSTEM DESIGN}

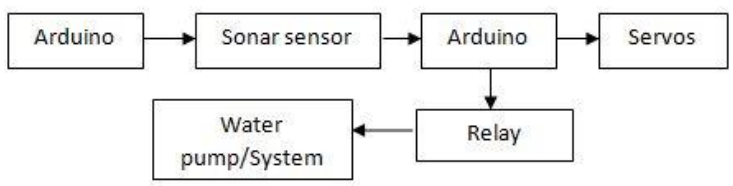

Fig 1. Block Diagram of the System

\section{A. Arduino Uno}

Arduino/Genuino Uno is addressed as a microcontroller board established on the ATmega328P Chip. It consists 14 digital Output/Input pins among which 6 are used as PWM outputs, another 6 are used as inputs. There is also a 16 $\mathrm{MHz}$ quartz crystal, one USB connection, one power jack, one ICSP header and one reset button is inserted in the board. It carries everything that is needed to support the microcontroller. It can be power up by using the USB cable or AC-DC adapter or a battery [7].

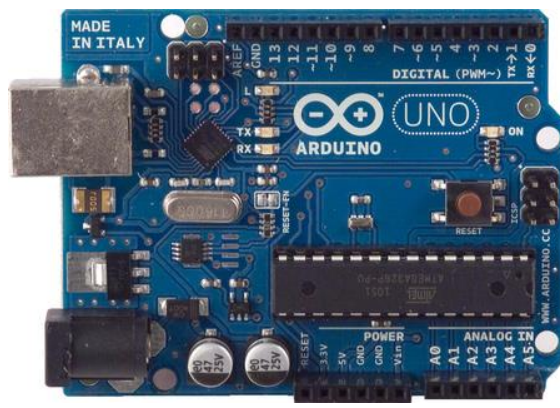

Fig 2: Arduino UNO

\section{B. SG90 Micro Servo}

The SG90 Micro Servo motors are small and lightweight with high output power. They can rotate $180^{\circ}\left(90^{\circ}\right.$ in both direction), and works just same as the standard servos but smaller in size. Any arduino build in code for servo, hardware or library can be labeled for specific performance. It comes with a 3 horns (arms). Its stall torque is $1.8 \mathrm{kgf.cm}$ and running speed is $0.1 \mathrm{~s} / 60$ degree [8]

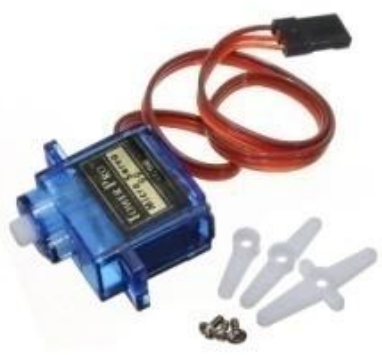

Fig.3. SG90 Micro Servo

\section{HC-SR-04 Sonar Sensor}

The HC-SR-04 ultrasonic sensor operates with the same principal that bat and dolphin's uses to locate an object. Ultrasonic sensor uses sound propagation to calculate distance between sensor and object. It has some excellent features such as zero-contact range detection with high accuracy and reliable data reading. It has calmness operating range of $2 \mathrm{~cm}$ to $400 \mathrm{~cm}$ or 1 " to 13 feet. Sunlight or black material (such as Sharp rangefinders) can't resist its operation. Ultrasonic transmitter and receiver complete the HC-SR-04 Ultrasonic sensor module [9].

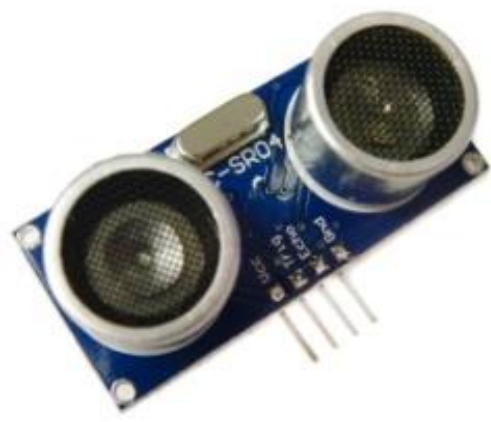

Fig.4. HC-SR 04 Sonar sensor

\section{L293D DC Motor Driver}

L293D is a motor driver Integrated Circuit which goes after dual H-bridge principal. It receives a low-current control signal as input and gives a higher-current output signal which drives the dc motors. Maximum two dc motors can be driven at the same time in both forward and reverse direction under normal operating condition. Input logics are arranged at Pin $2 \& 7$ and $10 \& 15$ to drive the motors When input logic is 00 or 11 the corresponding motor will stop [10].

\section{E. HS-5805 Mega Servo (Metal Gear)}

The HS-5805MG is the digital kinfolk of the HS-805MG and just as the HS-805MG it was designed to operate the punishment dished out by the current wave of large-scale r/c conveyance. Using of the digital circuit, include huge holding torque and the capability to program the servo. It has a no-load speed $0.19 \mathrm{sec} / 60$ and voltage range $4.8 \mathrm{~V}$ $6.0 \mathrm{~V}$. It has four metal gears and one heavy duty Nylon gear [11].

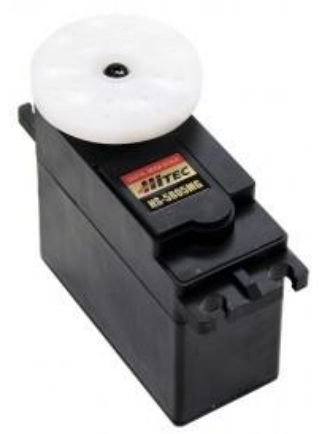

Fig.5. HS-5805 Mega Servo

\section{F. Water Pump}

This mini Submersible water pump needs DC12V 4.8W to run. It is usually used in Fish Tank Aquarium Fountain Flowerpot. This kind pump consists a brushless DC motor. Its biggest advantage is it has a low consumption but having a long working life [12]. 
IV. One Dimension Design

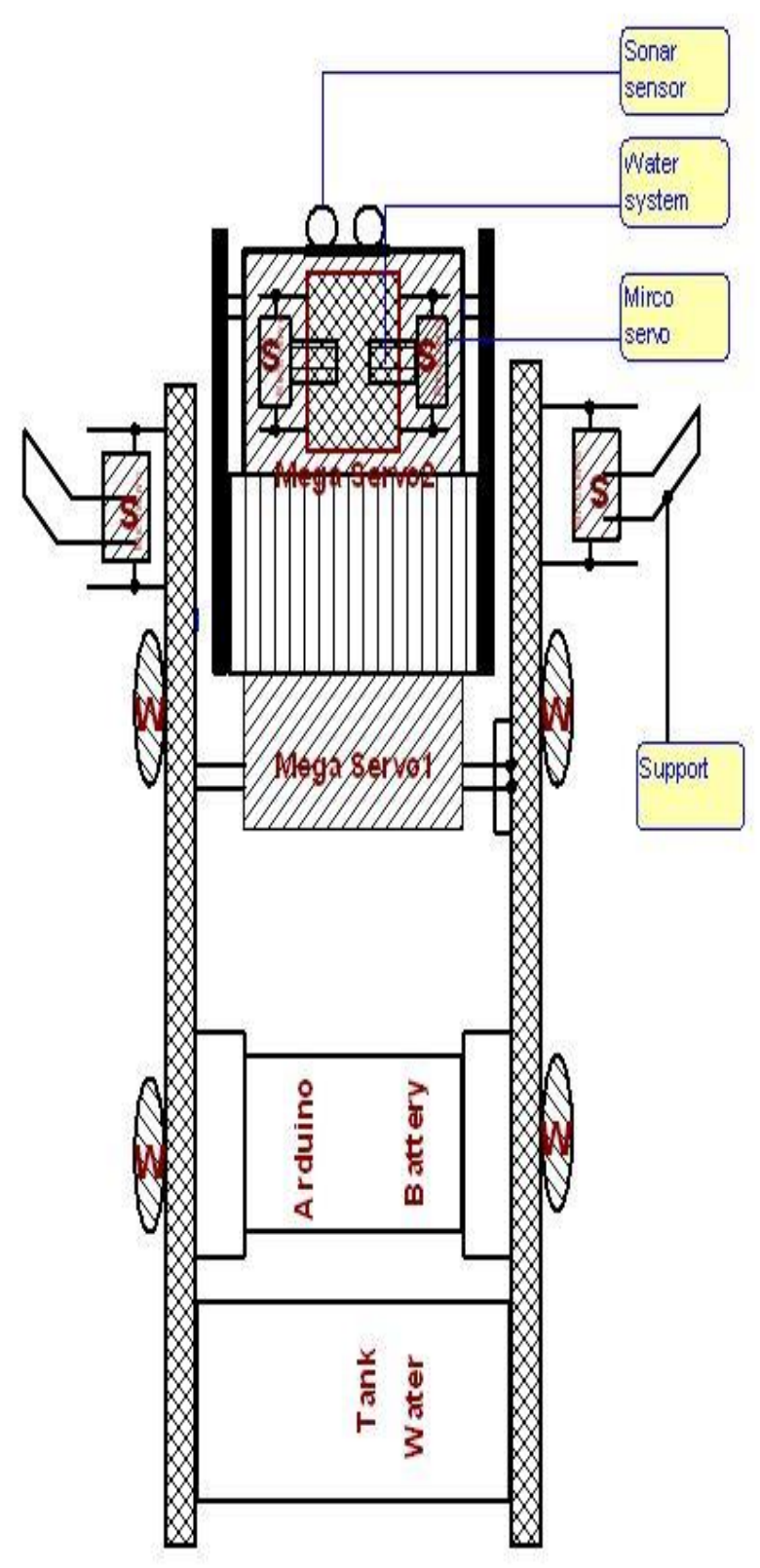

V. FLOW CHART

A. Automatic Transformation

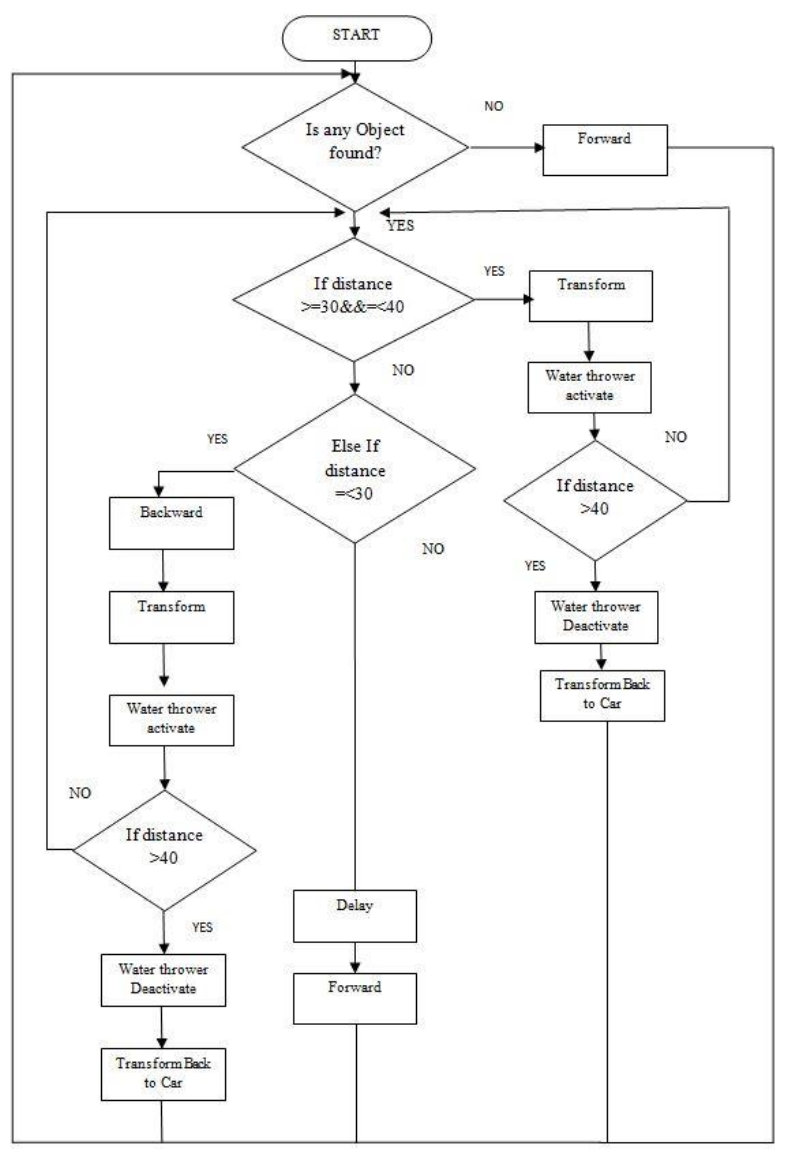

Fig.7. Flow chart for Automatic Transformation

Fig.6. One D design of the robot 


\section{B. Manual Transformation}

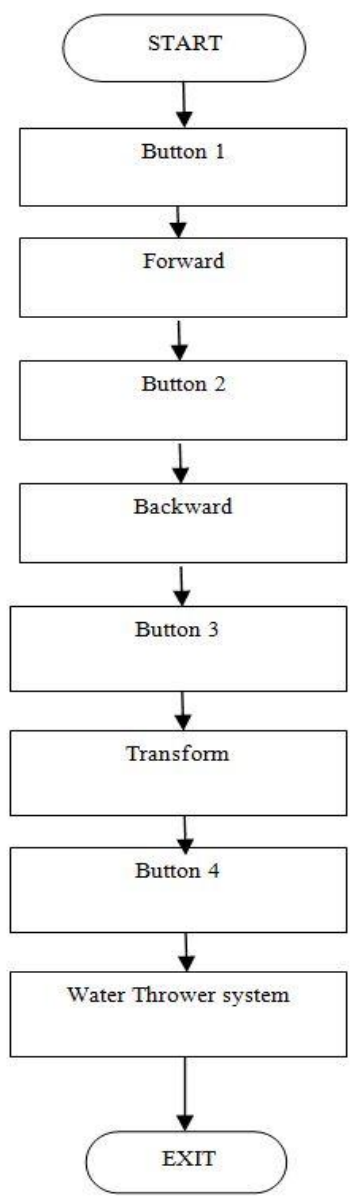

Fig.8. Flow chart for Manual Transformation

\section{CONCLUSION}

In this paper, a robot that can transform and also implemented a water thrower system for full-filling its purpose is designed. The whole process depends on the sonar sensor and how it response according to the logics. Use of sonar sensor makes the robot self-intelligence. For minimizing the overall cost, nearby available equipment and grazes are considered to build the robot and as it's a demo model fish tank's water pump is used for the water thrower system. The further selection of the characteristic and specification will build upon shape, artistry and control methods.

The proposed model can be used for resisting violence, rescue missions, defend attacks and in the workplaces that is hard or dangerous for law enforcement officers.

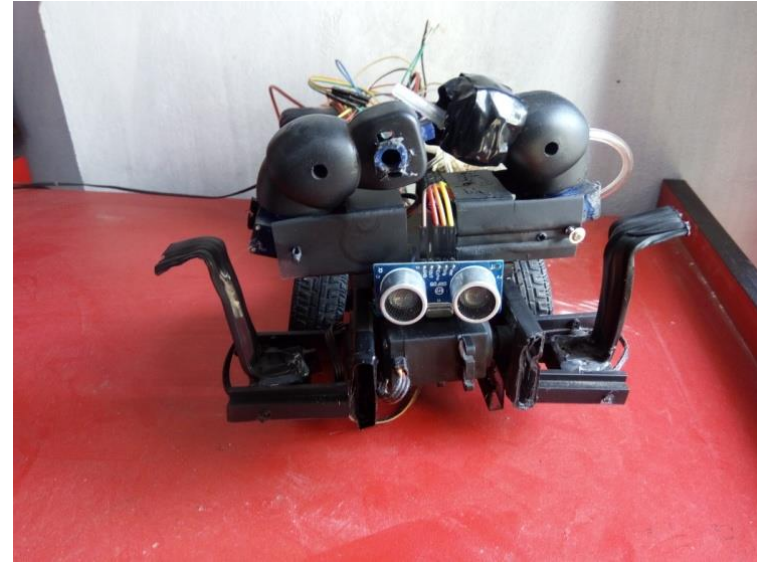

Fig.9. Two dimensional View of Car Formation

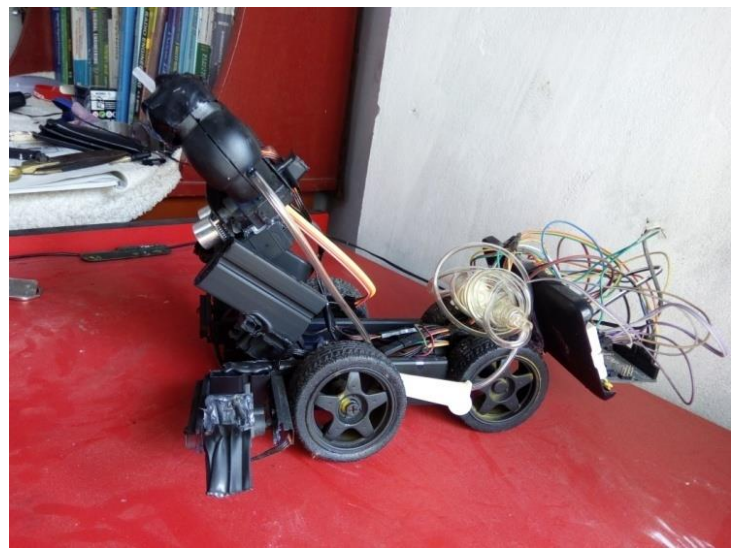

Fig.10. Two dimensional View of Transforming stage

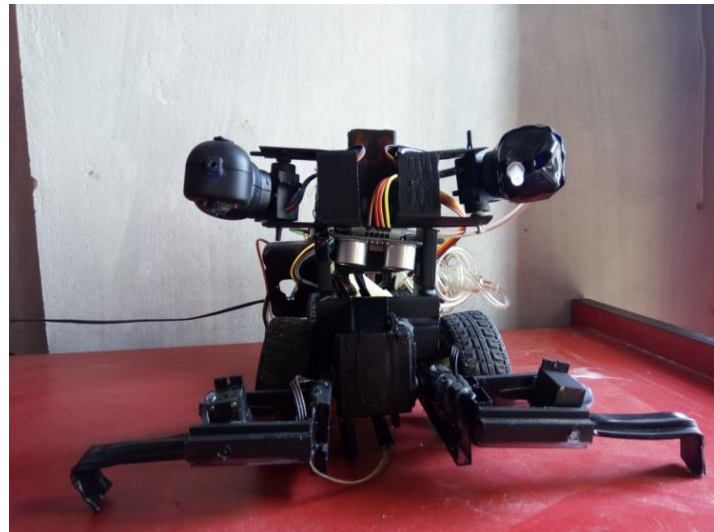

Fig.11. Two dimensional View of Fully Transformed Robot with water thrower system

\section{REFERENCES}

[1] Available:

http://www.engpaper.com/free-research-papers-and-projects-onrobotics.htm

[2] S.K.Mostaque, B.Karmakar, "Low Cost Arduino Based Voice Controlled Pick and Drop Service with Movable Robotic Arm". European Journal of Engineering Research and Science, Vol 1(5), November, 2016.

[3] Available: https://www.theguardian.com/technology/2016/jul/08/police-bombrobot-explosive-killed-suspect-dallas.

[4] Available: http://www.learnaboutrobots.com/lawEnforcement.htm

[5] K.R.Chaubey, T.Singh, S.Jasuja, "Leg to Wheel and Wheel to Leg Transformer Robot having ability of Obstacle Detection and Controlled by Cell Phone using DTMF Technique", Asia Pacific Institute of Information Technology, India. Available: https://www.researchgate.net/project/Leg-to-wheel-and-Wheel-toLeg-Transformer-Robot?openDialog=followers

[6] Available: 
https://www.arduino.cc/en/Main/ArduinoBoardUno

[7] SG90-Datasheet,Available:

http://www.micropik.com/PDF/SG90Servo.pdf.

[8] HC-SR04 Ultrasonic Sensor User's Manual, Cytron Technologies. Available:

https://docs.google.com/document/d/1YyZnNhMYy7rwhAgyL_pfa39RsB-x2qR4vP8saG73rE/edit

[9] L293D-Datasheet,Available:

http://www.engineersgarage.com/electronic-components/1293dmotor-driver-ic.

[10] Available:

https://www.servocity.com/hs-5805mg-servo

[11] Available:

http://www.ebay.com/itm/Mini-DC12V-4-8W-240L-H-BrushlessSubmersible-Aquarium-Fish-Tank-Water-Pump-2H5Z-

/151789545536

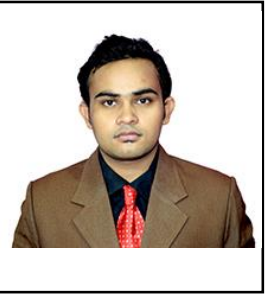

Bishal karmakar comes from Bangladesh .His date of birth is 30.09.1995. He is currently doing his final year of B.Sc. in Electrical and Electronic Engineering from Varendra University, Rajshahi, Bangladesh.

$\mathrm{He}$ is been working on robotics for two years. He is mentoring the students of E-club on Basic Robotics, Arduino Programming and Embedded Design in Varendra University, Rajshahi,

Bangladesh

Mr.Karmakar has earned the $1^{\text {st }}$ runner up position in Robodroid Championship, 2016 arranged by IEEE in RUET, among all the teams from different universities of Bangladesh. 